\title{
Phase-Dependent Molecular Requirements for Memory Reconsolidation: Differential Roles for Protein Synthesis and Protein Kinase A Activity
}

\author{
György Kemenes, ${ }^{1}$ Ildikó Kemenes, ${ }^{1}$ Maximilian Michel, ${ }^{1}$ Andrea Papp, ${ }^{1}$ and Uli Müller ${ }^{2,3}$ \\ ${ }^{1}$ Sussex Centre for Neuroscience, Department of Biology and Environmental Sciences, School of Life Sciences, University of Sussex, Falmer, Brighton BN1 \\ 9QG, United Kingdom, ${ }^{2}$ Institut für Biologie-Neurobiologie, Freie Universität Berlin, D-14195 Berlin, Germany, and ${ }^{3}$ Department 8.3 Biosciences-Zoology/ \\ Physiology, Natural Sciences and Technology III, Saarland University, D-66041 Saarbrücken, Germany
}

\begin{abstract}
After consolidation, a process that requires gene expression and protein synthesis, memories are stable and highly resistant to disruption by amnestic influences. Recently, consolidated memory has been shown to become labile again after retrieval and to require a phase of reconsolidation to be preserved. New findings, showing that the dependence of reconsolidation on protein synthesis decreases with the age of memory, point to changing molecular requirements for reconsolidation during memory maturation. We examined this possibility by comparing the roles of protein synthesis (a general molecular requirement for memory consolidation) and the activation of protein kinase A (PKA) (a specific molecular requirement for memory consolidation), in memory reconsolidation at two time points after training. Using associative learning in Lymnaea, we show that reconsolidation after the retrieval of consolidated memory at both 6 and $24 \mathrm{~h}$ requires protein synthesis. In contrast, only reconsolidation at $6 \mathrm{~h}$ after training, but not at $24 \mathrm{~h}$, requires PKA activity, which is in agreement with the measured retrieval-induced PKA activation at $6 \mathrm{~h}$. This phase-dependent differential molecular requirement for reconsolidation supports the notion that even seemingly consolidated memories undergo further selective molecular maturation processes, which may only be detected by analyzing the role of specific pathways in memory reconsolidation after retrieval.
\end{abstract}

Key words: classical conditioning; memory retrieval; memory reconsolidation; PKA; protein synthesis; Lymnaea

\section{Introduction}

Memories are highly dynamic and exist in several phases, often characterized by the different molecular processes required for their induction or maintenance (for review, see Abel and Lattal, 2001; Kandel, 2001). The process of memory consolidation requires translation and transcription, but when memories become consolidated, they are no longer sensitive to amnestic treatments with transcription and translation blockers (Davis and Squire, 1984). A number of reports have now convincingly shown that after the retrieval of consolidated memories, the memory trace undergoes an active process called reconsolidation, which, like consolidation itself, can be interrupted by amnestic treatments (Dudai and Morris, 2000; Nader et al., 2000; Sara, 2000; Nader, 2003). However, another emerging notion is that the susceptibility of consolidated memory to disruptions after retrieval might decrease with the age of the memory (Alberini, 2005). For example, recent work showed that the requirement for protein synthe-

Received Feb. 28, 2006; revised May 2, 2006; accepted May 3, 2006.

This work was supported by the Royal Society (UK; G.K. and U.M.), the Medical Research Council (UK; G.K.), the Biotechnology and Biological Sciences Research Council (UK; G.K.), and the Deutsche Forschungsgemeinschaft (Germany; U.M.).

Correspondence should be addressed to Prof. György Kemenes, Sussex Centre for Neuroscience, School of Life Sciences, Department of Biology and Environmental Sciences, University of Sussex, Falmer, Brighton BN1 90G, UK. E-mail:G.Kemenes@sussex.ac.uk.

DOI:10.1523/JNEUROSCI.0890-06.2006

Copyright $\odot 2006$ Society for Neuroscience $\quad$ 0270-6474/06/266298-05\$15.00/0 sis for reconsolidation diminishes as consolidated memories get older (Milekic and Alberini, 2002; Lattal and Abel, 2004). This finding raises the interesting possibility that consolidated memory also has different phases (e.g., freshly consolidated and older), indistinguishable by amnestic treatments without retrieval but identifiable by their changing molecular requirements for reconsolidation.

We tested this hypothesis by directly comparing the function of protein synthesis and protein kinase A (PKA) activation in the reconsolidation process in the snail Lymnaea stagnalis, an experimentally tractable molluscan model system for studying the mechanisms of learning and memory (Benjamin et al., 2000). Lymnaea builds a robust associative long-term memory (lasting for $>2$ weeks) after reward classical conditioning using a single pairing of a chemical stimulus, amyl acetate, with a food stimulus, sucrose (Alexander et al., 1984). The use of this single-trial paradigm for the analysis of the molecular mechanisms of memory reconsolidation has two main advantages. First, after single-trial conditioning, translation-dependent memory emerges in a matter of hours (Fulton et al., 2005), allowing the reconsolidation of translation-dependent memory to be studied on a timescale of a few hours to a few days. Second, unlike multitrial paradigms, it allows the amnestic effects of sharply timed manipulations of key molecular pathways to be analyzed (Kemenes et al., 2002; Fulton et al., 2005; Ribeiro et al., 2005).

We focused on PKA as a specific signaling molecule because 
although it is known to play a highly conserved key role in longterm memory formation (Drain et al., 1991; Huang et al., 1994; Müller, 2000), evidence for its role in memory reconsolidation has just started to emerge (Tronson et al., 2006). By both directly measuring retrieval-induced PKA activation and showing that it is necessary for memory reconsolidation, we first demonstrate phase-dependent differences between the effects of postretrieval inhibition of protein synthesis and PKA activity on memory reconsolidation. Our observations indicate that depending on the age of the consolidated memory, different molecular pathways are activated by memory retrieval and contribute differentially to memory reconsolidation.

\section{Materials and Methods}

Experimental animals and chemicals

A laboratory-bred stock of Lymnaea stagnalis was maintained before the learning experiments, as described previously (Kemenes et al., 2002). All chemicals were from Sigma-Aldrich (Poole, Dorset, UK) unless stated otherwise.

\section{Single-trial conditioning protocol}

Appetitive (food-reward) chemical classical conditioning of intact Lymnaea was performed using established methods based on single-trial training, control, and handling protocols, described in detail in previous publications (Alexander et al., 1984; Kemenes et al., 2002; Ribeiro et al., 2003, 2005; Straub et al., 2004; Fulton et al., 2005). The conditioned stimulus (CS) was amyl acetate (final concentration, $0.004 \%$ ), and the unconditioned stimulus (US) was sucrose (final concentration, $0.67 \%$ ). The CS-US interval was $15 \mathrm{~s}$ in the paired group and $1 \mathrm{~h}$ in the unpaired control group.

\section{In vitro assay of PKA activity induced in vivo}

Animals were individually trained (CS/US paired protocol) or subjected to a control protocol (CS/US unpaired) using a staggered experimental regimen to allow each animal to be killed at a precise time point after single-trial training or control treatment and subsequent memory retrieval. Cerebral ganglia were dissected quickly $(<30 \mathrm{~s})$ on ice and immediately homogenized in $100 \mu \mathrm{l}$ of micropipette tubes (Blaubrand, Wertheim, Germany), each containing $20 \mu \mathrm{l}$ of frozen homogenization buffer (in mm: 50 Tris-HCl, pH 7.7, 10 2-mercaptoethanol, 1 EDTA, and 1 EGTA). All samples were stored in liquid $\mathrm{N}_{2}$ before subjecting them to an in vitro PKA assay used previously to determine PKA activity induced in vivo in honeybees and Aplysia (Müller and Carew, 1998; Müller, 2000).

\section{Amnestic treatments and memory tests}

Inhibiting translation after memory retrieval. To test the amnestic effects of inhibiting translation, a batch of snails was conditioned with a single CS/US pairing and randomly divided into four groups. Snails in two of the four groups received a single application of the CS $6 \mathrm{~h}$ after training (memory retrieval). Ten minutes after memory retrieval, the snails in one of these two groups were injected with $1 \mathrm{~mm}$ anisomycin, while the snails in the second group were injected with saline. The drug and control solutions were injected into the heamocoel directly surrounding the central ganglia. Snails in the remaining two groups received no CS application at $6 \mathrm{~h}$ (no memory retrieval) but were injected either with anisomycin or saline $6 \mathrm{~h}, 10 \mathrm{~min}$ after training. Twenty-four hours after training, snails in all four conditioned groups were retested for their feeding responses to the CS.

To test the effect of inhibiting translation after later memory retrieval, the snails were treated similarly to the $6 \mathrm{~h}$ experiment but instead memory retrieval (or no retrieval) took place at $24 \mathrm{~h}$, and anisomycin or saline was administered $10 \mathrm{~min}$ later. Similar to the previous experiment, the snails in this experiment were retested for their feeding response to the CS $18 \mathrm{~h}$ after reactivation ( $42 \mathrm{~h}$ after training).

Inhibiting PKA activity after memory retrieval. The experiments concerning the amnestic effects of inhibiting PKA activity at different posttraining time points, with or without memory retrieval, were designed in a similar way to the experiments addressing the amnestic effects of inhibiting translation. The amnestic agent used was KT5720 (Alomone Labs,
Jerusalem, Israel), a specific PKA inhibitor, at $10 \mu \mathrm{M}$ in $0.5 \%$ DMSO (final concentrations), whereas the vehicle was a mixture of saline and $0.5 \%$ DMSO.

In both the translation and PKA inhibition experiments at each time point, handled snails were tested with the CS alongside the CS/US paired snails to obtain baseline CS feeding response levels. After the CS tests, all snails were also tested with the sucrose US to assess their ability to produce the basic feeding motor pattern. All tests were performed blind with the experimenter unaware of the treatment that each snail had undergone.

\section{Statistical analysis}

Comparisons between two groups were made using unpaired $t$ tests. Multiple comparisons were made using one-way ANOVA followed by Tukey's honestly significant difference post hoc tests. The effects of more than one factor on multiple groups were analyzed using two-way ANOVAs. Differences were considered significant at $p<0.05$. All detailed test statistics are shown in the supplemental material (available at www.jneurosci.org).

\section{Results}

\section{Reconsolidation of both fresh and older consolidated memory requires protein synthesis}

Previous experiments showed that in Lymnaea, memory expression $>5 \mathrm{~h}$ after single-trial classical reward conditioning requires a single time window of new protein synthesis shortly (between $10 \mathrm{~min}$ and $1 \mathrm{~h}$ ) after training (Fulton et al., 2005). Here, to investigate the reconsolidation of memory, which is consolidated with respect to early translation dependence but might have different phases distinguishable by retrieval-activated mechanisms, we examined both an early $(6 \mathrm{~h})$ and a later $(24 \mathrm{~h})$ time point after training. The $6 \mathrm{~h}$ memory was regarded as fresh, whereas the $24 \mathrm{~h}$ memory was regarded as older consolidated memory.

Injection of anisomycin at either $6 \mathrm{~h}$ (Fig. 1A) (Fulton et al., 2005 ) or $24 \mathrm{~h}$ after training (Fig. $1 B$ ) had no effect on later memory retrieval (Fig. $1 A, 24 \mathrm{~h} \mathrm{CS}, B, 42 \mathrm{~h} C S$ ), confirming that memory at both time points can be considered as consolidated. Moreover, injection of anisomycin after memory retrieval (Fig. $1 A, B, \mathrm{CS}$ ) at either $6 \mathrm{~h}$ (Fig. $1 A$ ) or $24 \mathrm{~h}$ (Fig. $1 B$ ) led to subsequent impairment of memory. With regard to the function of translation blockers, this is in agreement with findings in other systems (Sara, 2000; Abel and Lattal, 2001; Nader, 2003; Dudai and Eisenberg, 2004) and also with findings using another associative learning paradigm (operant conditioning) in Lymnaea itself, which first showed translation-dependent reconsolidation in this species (Sangha et al., 2003). Thus, our finding supports the general concept that memory considered to be consolidated with respect to translation becomes sensitive to translational blockers after retrieval.

\section{Retrieval of consolidated memory activates PKA in a phase-dependent manner}

In several systems, inhibition experiments have indicated that memory retrieval may activate various signaling cascades, which are required for subsequent memory expression (Dudai and Eisenberg, 2004; Alberini, 2005). Although the retrieval-induced activation of ERK (extracellular signal-regulated kinase) and a number of immediate-early genes has been shown before (Hall et al., 2001; Tronel and Sara, 2002; Kelly et al., 2003; Strekalova et al., 2003; von Hertzen and Giese, 2005), no data were available with regard to retrieval-induced activation of PKA. Therefore, we directly measured PKA activity shortly after memory retrieval at 6 or $24 \mathrm{~h}$ after training, by using a technique applied previously to monitor training-induced PKA activation (Müller and Carew, 1998; Müller, 2000). Animals were subjected to a CS/US paired or 
A

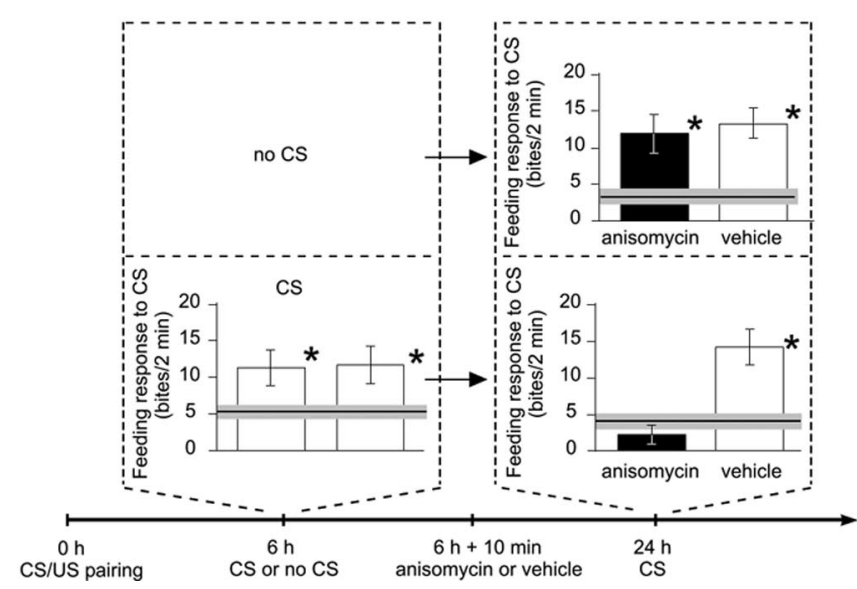

B

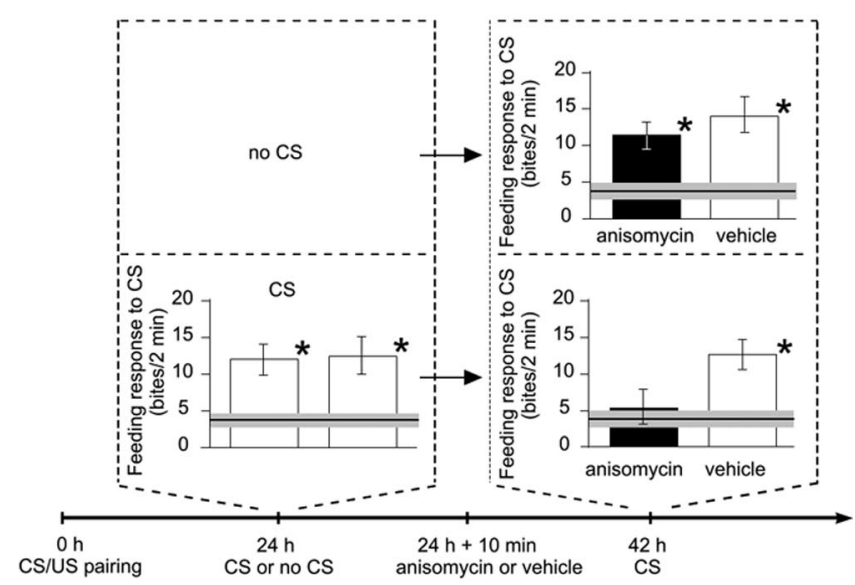

Figure 1. Protein synthesis is required after memory retrieval at both 6 and $24 \mathrm{~h}$ after training for later memory expression. Six hours $(\boldsymbol{A})$ or $24 \mathrm{~h}(\boldsymbol{B})$ after a single $C S / U S$ pairing $(0 \mathrm{~h})$, animals received a CS stimulation or no stimulation (no CS), followed 10 min later by injection with either the translation blocker anisomycin or vehicle. In each memory test, baseline levels are defined by the mean feeding response level to the CS (line) \pm SEM (gray band) of groups of animals handled in parallel $(n=17)$. ANOVAs followed by pairwise post hoc tests reveal that the groups first tested at $6 \mathrm{~h}(\boldsymbol{A})(n=19$ and 20$)$ and the groups first tested at $24 \mathrm{~h}(\boldsymbol{B})(n=20 \mathrm{each})$ show significantly $\left({ }^{*}\right)$ elevated feeding responses to the $C S$ compared with baseline level. Regardless of the injection, animals tested at $24 \mathrm{~h}(n=17$ and 19$)$ and $42 \mathrm{~h}(n=14$ and 18$)$ with no previous $(S$ test (no $C S)$ also show significant feeding responses compared with baseline. In contrast, the CS/US paired groups that were both given a retrieval trial (CS) at either 6 or $24 \mathrm{~h}$ after training and subsequently injected with anisomycin show feeding responses to the CS that are not significantly above baseline. Two-way ANOVAs comparing the feeding responses to the $C S$ of the four CS/US paired groups detected a significant interaction of memory retrieval and subsequent protein synthesis inhibition at both the $24 \mathrm{~h}(\boldsymbol{A})$ and $42 \mathrm{~h}(\boldsymbol{B})$ memory test. For the detailed test statistics for ANOVAs and pairwise comparisons, see the supplemental material (available at www.jneurosci.org).

CS/US unpaired training protocol. PKA activity in the cerebral ganglia was determined $5 \mathrm{~min}$ after a single CS application (memory retrieval in the conditioned group) or at the corresponding time point in the case of animals that received no CS (Fig. 2A). The cerebral ganglia were targeted in these experiments because they are important sites for neuronal plasticity induced by singletrial reward conditioning (Straub et al., 2004).

At the behavioral level, we found robust memory retrieval in the CS/US paired groups at both 6 and $24 \mathrm{~h}$ (Fig. 2B). Biochemical measurements using a PKA-specific substrate revealed a significant elevation of PKA activity in the CS/US paired group after memory retrieval at $6 \mathrm{~h}$ after training compared with both the two CS/US unpaired control groups and the CS/US paired group in which the memory was not retrieved (Fig. 2Ci). In contrast, memory retrieval at $24 \mathrm{~h}$ after training did not elevate PKA activity in any of the groups (Fig. 2Cii). These experiments provide the first direct evidence for phasedependent retrieval-induced PKA activation.

\section{Inhibition of retrieval-induced PKA activity impairs the reconsolidation of fresh consolidated memory}

To verify the function of the observed retrieval-induced PKA activation, we performed blocking experiments after memory retrieval. In a first experiment, we confirmed that injection of the PKA inhibitor KT5720 without memory retrieval (Fig. $3 A, B$, no CS) at either $6 \mathrm{~h}$ (Fig. $3 A$ ) or $24 \mathrm{~h}$ after training (Fig. $3 B$ ) had no effect on later memory retrieval. Thus, at either 6 or $24 \mathrm{~h}$ after conditioning, PKA activity is not essential for ongoing processes of memory formation or memory maintenance. However, retrieval of memory followed by inhibition of PKA leads to differential effects on subsequent memory performance, depending on the time after training. Figure $3 A$ shows that memory retrieval at $6 \mathrm{~h}$ after training followed by KT5720 injection leads to a significant reduction in the conditioned response tested at $24 \mathrm{~h}$. Inhibition of PKA shortly after memory retrieval at $24 \mathrm{~h}$ did not affect
A

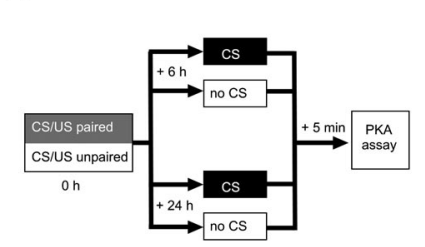

B

$\mathrm{Ci}$

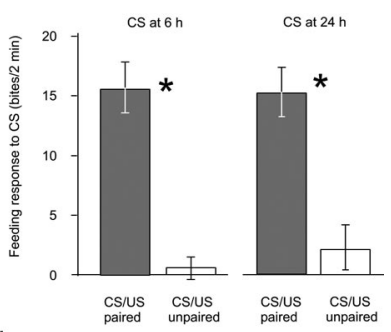

Cii
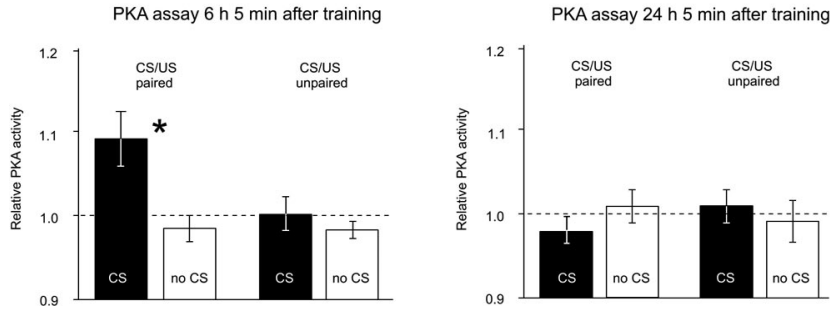

Figure 2. Memory retrieval at 6 but not $24 \mathrm{~h}$ after training activates PKA. A, A simplified diagram of the experiment comprising CS/US paired or CS/US unpaired training (0 h) [for more details, see Kemenes et al. (2002)], posttraining treatment (CS or no $C S$ at 6 or $24 \mathrm{~h}$ after training), and PKA assays (5 min after the time point for $(S$ or no $C S) . B$, Memory retrieval by the presentation of the $C S$ at 6 and $24 \mathrm{~h}$ after training reveals significant $\left({ }^{*}\right)$ differences between the CS/US paired group ( $n=14$ each) and the CS/US unpaired group ( $n=15$ each). Ci, Cii, PKA activity measured $5 \mathrm{~min}$ after the presentation of $\mathrm{CS}$ at 6 or $24 \mathrm{~h}$ after training or at corresponding time points without the presentation of the $C S$ (no $C S)$. A two-way ANOVA reveals that memory retrieval at $6 \mathrm{~h}$ after training leads to a significant $\left({ }^{*}\right)$ increase in PKA activity selectively in the CS/US paired group. Memory retrieval at $24 \mathrm{~h}$ after training does not lead to an increase in PKA activity in any of the groups. Error bars represent SEM. For the detailed test statistics for ANOVAs and pairwise comparisons, see the supplemental material (available at www.jneurosci.org).

later memory (Fig. $3 B, 42 \mathrm{~h}$ ), which is in accordance with the absence of retrieval-induced PKA activation at $24 \mathrm{~h}$.

Next, we sought to determine whether it was the activation of the CS pathway or the resulting activation of the feeding motor 
A

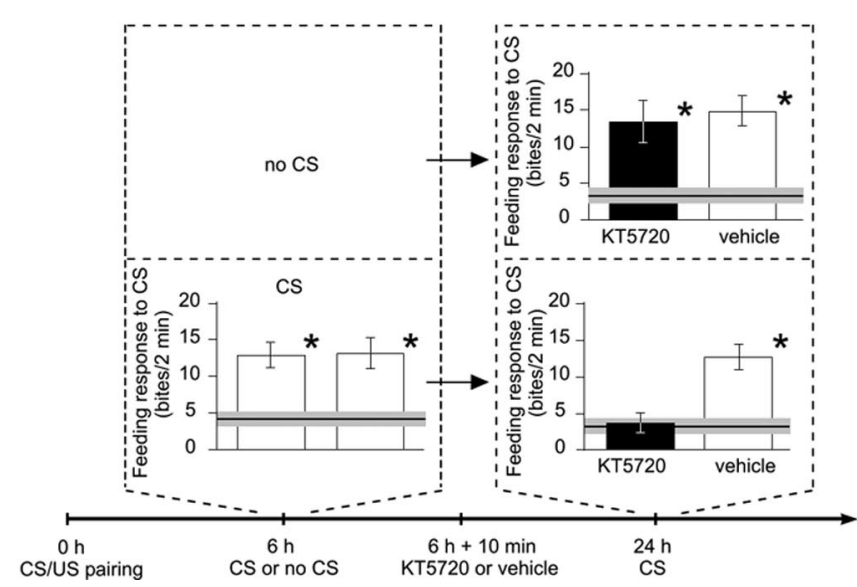

B

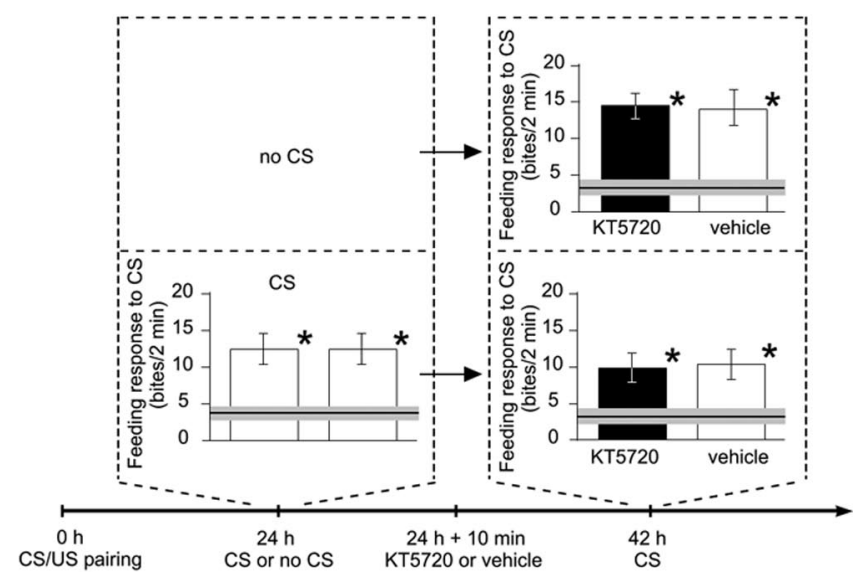

Figure 3. PKA activity is required after memory retrieval at 6 but not $24 \mathrm{~h}$ after training for later memory expression. Six hours $(\boldsymbol{A})$ or $24 \mathrm{~h}(\boldsymbol{B})$ after a single $C S / U S$ pairing $(0 \mathrm{~h})$, animals received a CS stimulation or no stimulation (no CS), followed 10 min later by an injection with either a PKA inhibitor (KT5720) or vehicle. In each group, baseline levels are defined by the mean feeding response level to the CS (line) \pm SEM (gray band) of a group of animals handled in parallel $(n=17)$. A, ANOVAs followed by pairwise post hoc tests reveal that the two groups ( $n=24$ and 21$)$ first tested at $6 \mathrm{~h}$ after training and $10 \mathrm{~min}$ before injection show significantly (*) elevated feeding responses to the CS compared with baseline level. At the second test, $24 \mathrm{~h}$ after training, one-way ANOVAs detected a source of significant difference. Of the four CS/US paired groups, only the group that received the CS at $6 \mathrm{~h}$ after training followed by KT5720 injection shows a feeding response to the CS that does not differ from baseline. A two-way ANOVA comparing the feeding responses to the $C S$ of the four CS/US paired groups at $24 \mathrm{~h}$ detected a significant interaction of memory retrieval at $6 \mathrm{~h}$ and subsequent PKA inhibition. $\boldsymbol{B}$, The two groups $(n=17$ and 20$)$ first tested at $24 \mathrm{~h}$ after training and $10 \mathrm{~min}$ before injection show significantly $(*)$ elevated feeding responses to the $(S \mathrm{~S} \mathrm{compared}$ with the baseline level. At $42 \mathrm{~h}$ after training, one-way ANOVAs and multiple post hoc comparisons reveal that all four CS/US paired groups show significantly stronger feeding responses to the CS than the handled groups against which they were compared but were not different from one another. A two-way ANOVA comparing the feeding responses to the CS of the four CS/US paired groups at $42 \mathrm{~h}$ detected no source of significant difference between the groups and no interaction of memory retrieval at $24 \mathrm{~h}$ and subsequent PKA inhibition. For the detailed test statistics for ANOVAs and pairwise comparisons, see the supplemental material (available at www.jneurosci.org).

program (Kemenes et al., 2002; Straub et al., 2004) that triggered subsequent PKA-dependent events required for memory reconsolidation. For these experiments, we used the same paradigm shown in Figure 3, but in this case presented an unconditioned feeding stimulus (sucrose) instead of the CS (amyl acetate) to conditioned animals at $6 \mathrm{~h}$ after training. In this case, we found that PKA inhibition did not abolish the conditioned response at the $24 \mathrm{~h}$ memory test (supplemental Fig. 1, available at www. jneurosci.org as supplemental material). Thus, postretrieval activation of the neuronal network underlying the feeding motor program (Benjamin et al., 2000) alone is not sufficient to make later memory expression dependent on PKA activation. Rather, it appears to be specifically the activation of the CS pathway that sets up the dependency on PKA activation required for memory reconsolidation.

\section{Discussion}

Whereas other studies on the molecular processes underlying reconsolidation have mainly focused on consolidated memories a long time (days to weeks) after training (Nader et al., 2000; Abel and Lattal, 2001; Milekic and Alberini, 2002), here we investigated reconsolidation processes in an earlier time window (6-24 h) after acquisition. In this earlier time window of consolidated memory, we identified phase-dependent differences in the requirement for protein synthesis and PKA activity for memory reconsolidation after retrieval. Our study shows that translationdependent processes are required for reconsolidation at both 6 and $24 \mathrm{~h}$. This supports the notion that translation-dependent processes are critical for reconsolidation independently of the time of retrieval, at least within the time window investigated here. However, this is not the case for the function of PKA in reconsolidation in the same time window. Our direct measurements show that memory retrieval at $6 \mathrm{~h}$ after training, but not at
$24 \mathrm{~h}$, activates PKA. In agreement with this, PKA inhibition directly after memory retrieval at $6 \mathrm{~h}$, but not at $24 \mathrm{~h}$, results in later memory impairment.

We also show that, without the application of the amyl acetate $\mathrm{CS}$, the activation of the motor program underlying the conditioned behavior cannot trigger subsequent PKA-dependent events required for memory reconsolidation, suggesting that the activation of the CS pathway is important for this process. The existence of an amyl acetate-activated CS chemosensory pathway in Lymnaea, which is electrophysiologically distinguishable from the sucrose-activated US pathway, has been demonstrated recently (Straub et al., 2004) and will need to be investigated with regard to its ability to activate PKA during memory retrieval.

A recent study by Tronson et al. (2006) demonstrated a bidirectional role for PKA in the reconsolidation of fear memory in the rat. Whereas blocking PKA activity in the basolateral amygdala after memory retrieval leads to impaired reconsolidation, activation of PKA after retrieval enhances reconsolidation. However, Tronson et al. (2006) did not investigate whether during retrieval the CS actually increased PKA activity. This was an important issue to clarify because the blocking of reconsolidation could also be caused by inhibition of baseline PKA activity while the CS activated other molecular cascades. By using a specific and sensitive PKA assay, our study is the first to provide direct evidence that PKA is activated during the retrieval of consolidated associative memory. Moreover, the use of this direct assay also helped us to establish that the retrieval of consolidated memory at a later time point does not activate PKA. These findings were corroborated by our findings from the postretrieval PKA inhibition experiments providing the first complete set of experimental evidence implicating retrieval-induced PKA activity in memory reconsolidation. 
Based on our findings, it also would be interesting to know whether the function of PKA in fear conditioning in mammals also reveals phase-dependent characteristics, and whether or not these are different from the phase-dependent requirements for protein synthesis. This, together with testing for a potential bidirectional function of PKA for reconsolidation in Lymnaea, would add substantially to our understanding of the conserved molecular mechanisms underlying reconsolidation.

Regardless of these comparative aspects, the demonstrated phase-dependent requirement for PKA activity for reconsolidation points to a complex retrieval-activated network of molecular processes required for memory reconsolidation, which may reflect ongoing processes of memory maturation ("lingering consolidation") (Dudai and Eisenberg, 2004). We previously showed nitric oxide (NO) to be required for memory consolidation for $\sim 5 \mathrm{~h}$ after training (Kemenes et al., 2002). Thus, for example, PKA activated by retrieval at $6 \mathrm{~h}$ after training might interact with some lingering NO-dependent consolidation process, albeit this interaction would occur at the tail end of the requirement for NO. An interaction between NO- and PKA-dependent memory consolidation processes has been found in the honeybee (Müller, 2000), and the same pathways may also interact in a brief overlapping period of lingering NO-dependent memory consolidation and PKA-dependent memory reconsolidation in the snail.

The finding that activation of a distinct signaling cascade by retrieval is restricted to a defined time window makes it likely that there are other pathways that behave in a similar manner in the same or different time windows. In agreement with other studies (for review, see Dudai and Eisenberg, 2004; Alberini, 2005), this finding also seems to rule out that reconsolidation is simply a recapitulation of consolidation, which could not be ruled out based on our protein-synthesis blocking experiments alone. Only a careful analysis by direct determination of various retrievalinduced molecular pathways in combination with blocking experiments will provide helpful information on the complex relationships between memory consolidation, retrieval-induced molecular processes, and their role in memory reconsolidation in different phases of consolidated memory.

\section{References}

Abel T, Lattal KM (2001) Molecular mechanisms of memory acquisition, consolidation and retrieval. Curr Opin Neurobiol 11:180-197.

Alberini CM (2005) Mechanisms of memory stabilization: are consolidation and reconsolidation similar or distinct processes? Trends Neurosci 28:51-56.

Alexander Jr J, Audesirk TE, Audesirk GJ (1984) One-trial reward learning in the snail Lymnaea stagnalis. J Neurobiol 15:67-72.

Benjamin PR, Staras K, Kemenes G (2000) A systems approach to the cellular analysis of associative learning in the pond snail Lymnaea. Learn Mem 7:124-131.

Davis HP, Squire LR (1984) Protein synthesis and memory: a review. Psychol Bull 96:518-559.

Drain P, Folkers E, Quinn WG (1991) cAMP-dependent protein kinase and the disruption of learning in transgenic flies. Neuron 6:71-82.

Dudai Y, Eisenberg M (2004) Rites of passage of the engram: reconsolidation and the lingering consolidation hypothesis. Neuron 44:93-100.

Dudai Y, Morris R (2000) To consolidate or not to consolidate: what are the questions? In: Brain, perception, memory. Advances in cognitive sciences (Bolhius J, ed), pp 149-162. Oxford: Oxford UP.

Fulton D, Kemenes I, Andrew RJ, Benjamin PR (2005) A single timewindow for protein synthesis-dependent long-term memory formation after one-trial appetitive conditioning. Eur J Neurosci 21:1347-1358.

Hall J, Thomas KL, Everitt BJ (2001) Cellular imaging of zif268 expression in the hippocampus and amygdala during contextual and cued fear memory retrieval: selective activation of hippocampal CA1 neurons during the recall of contextual memories. J Neurosci 21:2186-2193.

Huang Y-Y, Li XC, Kandel ER (1994) cAMP contributes to mossy fiber LTP by initiating both a covalently-mediated early phase and macromolecular synthesis-dependent late phase. Cell 79:69-79.

Kandel ER (2001) The molecular biology of memory storage: a dialogue between genes and synapses. Science 294:1030-1038.

Kelly A, Laroche S, Davis S (2003) Activation of mitogen-activated protein kinase/extracellular signal-regulated kinase in hippocampal circuitry is required for consolidation and reconsolidation of recognition memory. J Neurosci 23:5354-5360.

Kemenes I, Kemenes G, Andrew RJ, Benjamin PR, O'Shea M (2002) Critical time-window for NO-cGMP-dependent long-term memory formation after one-trial appetitive conditioning. J Neurosci 22:1414-1425.

Lattal KM, Abel T (2004) Behavioral impairments caused by injections of the protein synthesis inhibitor anisomycin after contextual retrieval reverse with time. Proc Natl Acad Sci USA 101:4667-4672.

Milekic MH, Alberini CM (2002) Temporally graded requirement for protein synthesis following memory reactivation. Neuron 36:521-525.

Müller U (2000) Prolonged activation of cAMP-dependent protein kinase during conditioning induces long-term memory in honeybees. Neuron 27:159-168.

Müller U, Carew TJ (1998) Serotonin induces temporally and mechanistically distinct phases of persistent PKA activity in Aplysia sensory neurons. Neuron 21:1423-1434.

Nader K (2003) Memory traces unbound. Trends Neurosci 26:65-72.

Nader K, Schafe GE, LeDoux JE (2000) Fear memories require protein synthesis in the amygdala for reconsolidation after retrieval. Nature 406:722-726.

Ribeiro MJ, Serfozo Z, Papp A, Kemenes I, O'Shea M, Yin JCP, Benjamin PR, Kemenes G (2003) Cyclic AMP response element-binding (CREB)-like proteins in a molluscan brain: cellular localization and learning-induced phosporylation. Eur J Neurosci 18:1223-1234.

Ribeiro MJ, Schofield MG, Kemenes I, O’Shea M, Kemenes G, Benjamin PR (2005) Activation of MAPK is necessary for long-term memory consolidation following food-reward conditioning. Learn Mem 12:538-545.

Sangha S, Scheibenstock A, Lukowiak K (2003) Reconsolidation of a longterm memory in Lymnaea requires new protein and RNA synthesis and the soma of right pedal dorsal 1. J Neurosci 23:8034-8040.

Sara SJ (2000) Retrieval and reconsolidation: toward a neurobiology of remembering. Learn Mem 7:73-84.

Straub VA, Styles BJ, Ireland JS, O'Shea M, Benjamin PR (2004) Central localization of plasticity involved in appetitive conditioning in Lymnaea. Learn Mem 11:787-793.

Strekalova T, Zorner B, Zacher C, Sadovska G, Herdegen T, Gass P (2003) Memory retrieval after contextual fear conditioning induces c-Fos and JunB expression in CA1 hippocampus. Genes Brain Behav 2:3-10.

Tronel S, Sara SJ (2002) Mapping of olfactory memory circuits: regionspecific c-fos activation after odor-reward associative learning or after its retrieval. Learn Mem 9:105-111.

Tronson NC, Wiseman SL, Olausson P, Taylor JR (2006) Bidirectional behavioral plasticity of memory reconsolidation depends on amygdalar protein kinase A. Nat Neurosci 9:167-169.

von Hertzen LS, Giese KP (2005) Memory reconsolidation engages only a subset of immediate-early genes induced during consolidation. J Neurosci 25:1935-1942. 